\title{
Precise fabrication of ultra violet dielectric dispersion compensating mirrors
}

\author{
T.Willemsen* ${ }^{\mathrm{a}, \mathrm{b}}$, S.Schlichting ${ }^{\mathrm{b}} ;$ T.Kellermann $^{\mathrm{b}} ;$ M.Jupé $^{\mathrm{b}} ;$ H.Ehlers $^{\mathrm{b}} ;$ U.Morgner $^{\mathrm{a}}$ and D.Ristau ${ }^{\mathrm{a}, \mathrm{b}}$ \\ a Institut für Quantenoptik, Leibniz University, QUEST, Welfengarten 1, 30167 Hanover, Germany; \\ ${ }^{\mathrm{b}}$ Laser Zentrum Hannover e.V., Hollerithallee 8, 30419 Hanover, Germany
}

\begin{abstract}
The present contribution is concentrated on an improved method to manufacture dielectric dispersion compensating mirrors in the ultra violet (UV) range by applying a novel online phase monitoring device. This newly developed measurement tool monitors the group delay (GD) and group delay dispersion (GDD) of the electromagnetic field in situ during the deposition of the layer system. Broad band monitoring of the phase enhances the accuracy in the near infrared spectral range (NIR), significantly. In this study, the correlation of the GDD in the NIR and in the UV spectral range is investigated. A design synthesis is introduced to achieve optimum reflection and GDD target values in the UV and NIR. This requires a similar behavior of both bands according to deposition errors, to guarantee switching off the UV GDD target band proper, while monitoring the GDD in the NIR spectral range. The synthesis results in a design, characterized by a GDD of $-100 \mathrm{fs}^{2} \pm 20 \mathrm{fs}^{2}$ between $330 \mathrm{~nm}$ and $360 \mathrm{~nm}$ in the UV and by $-450 \mathrm{fs}^{2} \pm 10 \mathrm{fs}^{2}$ within $820 \mathrm{~nm}$ to $870 \mathrm{~nm}$ in the NIR. The fabricated sample, applying an ion beam sputtering process, consists of a $9 \mu \mathrm{m}$ layer stack of Hafnium oxide and Silicon dioxide. The first layers of the stack are switched and controlled by a conventional in situ spectrometric broad band monitoring in conjunction with a forward re-optimization algorithm, which also manipulates the layers remaining for deposition at each switching event. To accomplish the demanded GDD-spectra, the last layers are controlled by the novel in situ GDD monitor.
\end{abstract}

Keywords: UV mirror, chirp mirror, IBS process, in situ measurement, GDD, design synthesis, ultra fast optics

\section{INTRODUCTION}

During the last years, ultra short pulse lasers (USP) gained more and more interest for medical and industrial applications. As a major aspect of USP laser applications, the natural dispersion of light or more precisely of USP propagating through a medium leads to a significant temporal pulse broadening. As a consequence, optical devices controlling the dispersion of light are necessary to ensure well defined temporal pulse shapes in commercial USPapplications. One promising component is the so called dispersion compensating mirror (DCM) [1]. Progresses in coating deposition techniques and material studies led to fabrications of DCMs covering a wavelength range of 4.5 octaves [2]. For example it is possible to generate laser pulses with a duration shorter than $4 \mathrm{fs}$ [3] in the visible range on the basis of dispersion compensating mirrors. Consequently, many commercial DCMs are available from the visible spectral range (VIS) up to the near infrared spectral range (NIR).

However the fabrication of ultra violet (UV) broadband dielectric dispersion compensating mirrors with high reflection is extremely challenging for wavelengths below $350 \mathrm{~nm}$. Only few materials with appropriately low optical absorption in the UV spectral range are available and the refractive index contrast between high and low index material is limited. For example, Hafnium oxide $\left(\mathrm{HfO}_{2}\right)$, Aluminum oxide $\left(\mathrm{Al}_{2} \mathrm{O}_{3}\right)$ and Silicon dioxide $\left(\mathrm{SiO}_{2}\right)$ are suitable materials, but the contrast between $\mathrm{Al}_{2} \mathrm{O}_{3}$ and $\mathrm{SiO}_{2}$ is very low. In addition, to achieve suitable GDD target values, many layers with a total thickness of several $\mu \mathrm{m}$ are required. Because of the increase in absorption with growing total thickness of the layer stack the reflection is reduced. Especially, in the UV range the losses of the coating materials are orders of magnitude higher than in the NIR or VIS spectral range, which intensifies the described problem even more. Considering the production, the average layer thickness of DCMs scales roughly linearly with the central wavelength, and consequently, the acceptable layer thickness tolerance tends to smaller values with shorter wavelength. Fabrication of DCMs for the VIS needs a layer thickness accuracy of $0.5 \mathrm{~nm}$. Extrapolating the central wavelength to the UV, a layer thickness precision of $0.1 \mathrm{~nm}$ is required [4], which leads to thickness precision and control of one atomic layer (e.g. the atomic radius of Hafnium is specified to $0.16 \mathrm{~nm}$ and of Silicon to $0.12 \mathrm{~nm}$, respectively).

Especially for the last layers of a DCM-stack, broad band monitoring (BBM), which is often employed in optical thin film deposition [5], reaches the limits for a precise and controlled fabrication. Consequently, the last layers are switched

*t.willemsen@1zh.de; phone +49 (0) 511 27788-294; fax +49 (0) 511 27788-100; www.lzh.de

Optical Systems Design 2015: Advances in Optical Thin Films V, edited by Michel Lequime,

H. Angus Macleod, Detlev Ristau, Proc. of SPIE Vol. 9627, 96271U · C 2015 SPIE

CCC code: $0277-786 \mathrm{X} / 15 / \$ 18 \cdot$ doi: $10.1117 / 12.2191051$

Proc. of SPIE Vol. $962796271 \mathrm{U}-1$ 
on time basis, which often requires several attempts to determine the precise deposition time of the sensitive layers. To reduce these limitations, another widely used technique is the fabrication of pairs of DCMs [6]. These mirrors are characterized by a well-defined shifted oscillating group delay dispersion (GDD), which lead to a compensated flat GDD after reflection by both mirrors. Compared to a single DCM, the production with an oscillating GDD is less sensitive to deposition errors.

However for the production of single DCMs, new monitor techniques are necessary for precise layer switching. As an approach, a novel monitor system is developed, which determines the group delay and the group delay dispersion in situ on moving substrates during complex coating processes. The setup consists of a fiber based Michelson white light interferometer and a fast spectrometer. On the basis of the components employed in this study, in situ group delay and GDD measurements can be performed in a spectral range from $800 \mathrm{~nm}$ up to $900 \mathrm{~nm}$ [7]. A new method is presented for the precise fabrication of ultra violet dielectric dispersion compensating mirrors applying the developed in situ phase measurement monitor system [7]. Depending on the specifications and the current measurement range of the phase monitor in the NIR and with respect to the group delay dispersion of two separated reflectance bands, special designs are calculated. The designs are adjusted in the measurement range of the phase monitor as well as optimized for the desired UV range according to the reflectance and GDD. Applying the phase monitor for a precise layer termination in the IRrange, the UV GDD band should be matched directly. Therefore designs are calculated which ensure an equal sensitivity to deposition errors for both bands. As an outcome a design is fabricated applying an ion beam sputter process. The UV band correlates to a band from $330 \mathrm{~nm}$ up to $360 \mathrm{~nm}$ with an average reflectivity of $94,5 \%$ and a GDD of $-100 \mathrm{ss}^{2} \pm 20 \mathrm{fs}^{2}$, respectively. In the first section the theoretical design considerations are presented, followed by a description of the deposition process and the results.

\section{DESIGN CONSIDERATIONS}

The presented design studies were preformed applying OptiLayer software [8]. In order to decrease the sensitivity to deposition errors during the manufacturing process, the robust synthesis procedure using needle optimization and gradual evolution algorithms was used for the design calculation [9]. The finally calculated and fabricated design consists of 78 alternating $\mathrm{HfO}_{2} / \mathrm{SiO}_{2}$ layers with a total physical thickness of $9.01 \mu \mathrm{m}$. Two target reflectance bands are specified. In the wavelength range between $330 \mathrm{~nm}$ up to $360 \mathrm{~nm}$, a group delay dispersion (GDD) of $-100 \mathrm{fs}^{2} \pm 20 \mathrm{fs}^{2}$ with an average reflectivity of $94.5 \%$ is realized. Concerning the second band of defined target points between $820 \mathrm{~nm}$ and $870 \mathrm{~nm}$, a GDD of $-450 \mathrm{fs}^{2} \pm 10 \mathrm{fs}^{2}$ and an average reflectivity of $97.5 \%$ is adjusted (see figure 1). It has to be mentioned that the reflectance band in the NIR is only addressed to monitor the GDD development during the deposition process. The band is not optimized with respect to a high reflectivity.

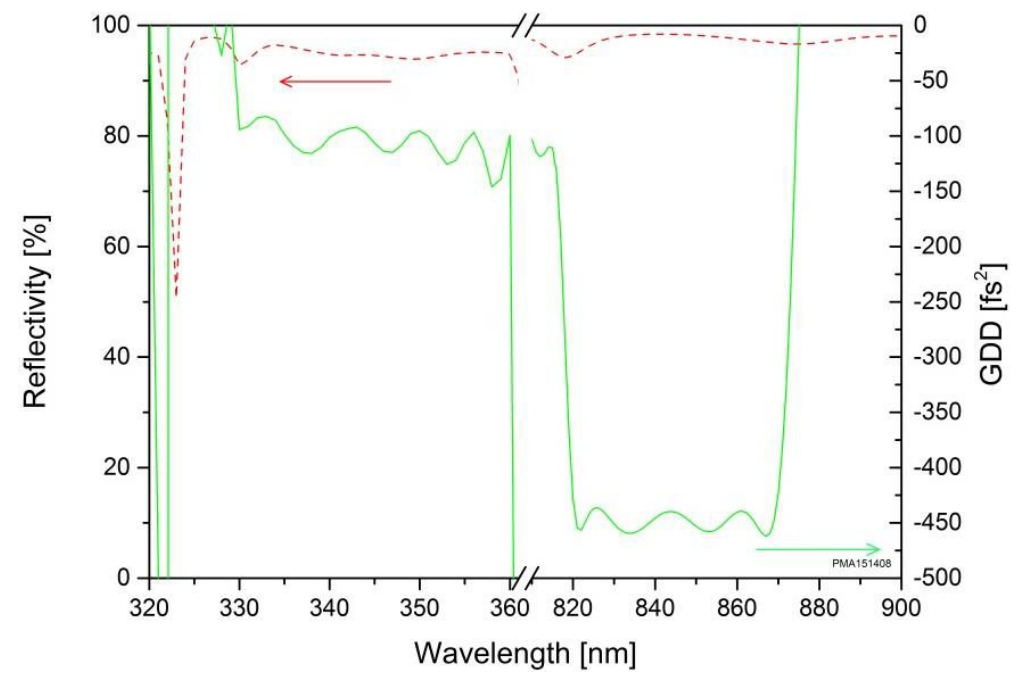

Figure1. Calculated reflectivity (dashed curve) and GDD (solid line). Within the target positions in the UV band $(330-360 \mathrm{~nm})$ the presented design has an average reflectivity of $94.5 \%$ and an average GDD of $-100 \mathrm{fs}^{2} \pm 20 \mathrm{fs}^{2}$, respectively. The reference target band $(820-870 \mathrm{~nm})$ is characterized by an average reflectivity of $97.5 \%$ and a GDD of $-450 \mathrm{fs}^{2} \pm 10 \mathrm{fs}^{2}$, respectively. 
Consequently, for ensuring a precise group delay dispersion of the fabricated mirror in the UV band, while monitoring the GDD in the NIR band, an equal sensitivity to deposition errors in both GDD target bands is required. Figure 2 plots the theoretic GDD and relating calculated changes due to an error in layer thickness of $\pm 1 \mathrm{~nm}$ in the last layer. Obviously, deposition errors affect the UV band more than the NIR band for a GDD ratio of 1:1 (UV:NIR) (see figure 2b). Considering the near linear scaling of layer thickness precision with central wavelength, a GDD ratio of 1:2 is expected, to range the sensitivity of both bands. However according to an empirical design study a ratio of approximately 1:4 has to be selected to provide a suitable thickness precision in the UV-range (see figure 2a). Additionally, further design synthesis indicates the possibility to shift the UV reflectance and GDD band closer to the DUV.

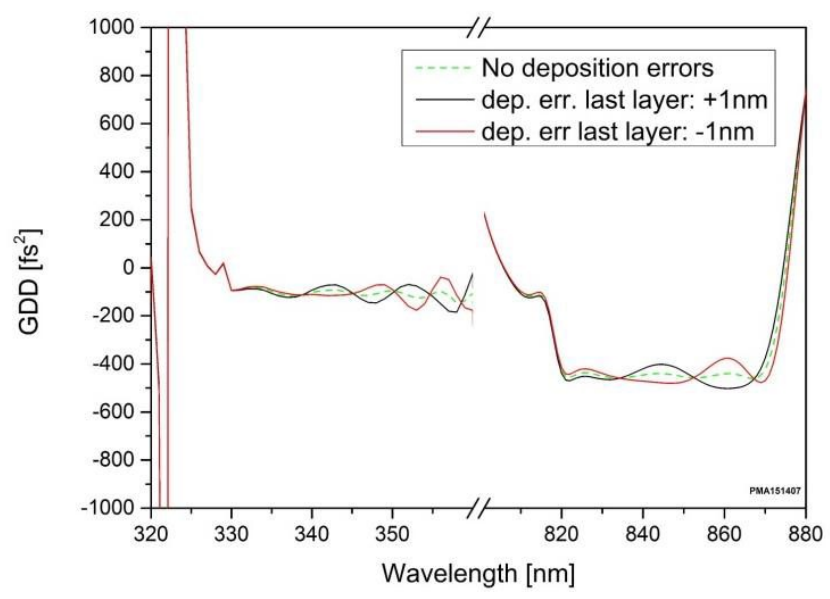

a) Calculated and fabricated design (HR850; -450fs ${ }^{2}$; HR355; -100fs $\left.{ }^{2}\right)$

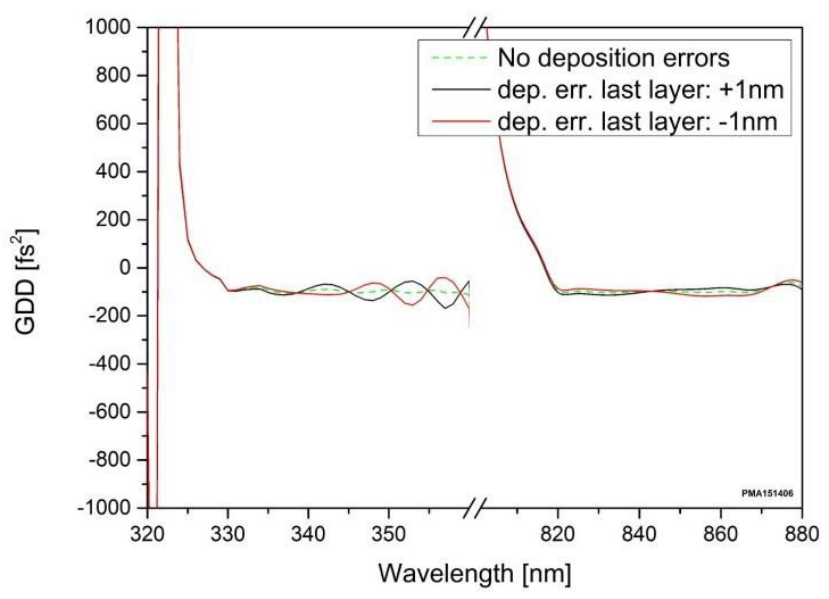

b) Calculated reference design (HR850; -100fs ${ }^{2} ;$ HR355; -100fs $\left.{ }^{2}\right)$

Figure 2. Sensitivity of the calculated GDD relating to deposition errors in the last layer of the particular design. The dashed curves represent the particular calculated design. The solid curves show the change of the GDD with a thickness error of $\pm 1 \mathrm{~nm}$ in the last layer, respectively.

\section{DEPOSITION PROCESS}

The mirrors were fabricated employing an ion beam sputtering process. The ion beam (Veeco $6 \mathrm{~cm}$ source, current $150 \mathrm{~mA}$, acceleration voltage $1500 \mathrm{~V}$ ) is extracted from a Xenon/Argon gas plasma by a three gird system and is directed on a flip target assembly, which is placed $40 \mathrm{~cm}$ from the source outlet and under an angle of incidence of 55 degree. As starting materials Silicon for the low refractive index and Hafnium for the high refractive index were selected for the target system, respectively. The layers are deposited in a reactive oxygen atmosphere of $8 \times 10^{-4} \mathrm{mbar}$ to achieve a balanced stoichiometry. $\mathrm{HfO}_{2}$ layers, characterized by a refractive index of 1.99 at a central wavelength of $\lambda_{0}=800 \mathrm{~nm}$, are sputtered with an average rate of $0.045 \mathrm{~nm} / \mathrm{s}$ and $\mathrm{SiO}_{2}\left(\mathrm{n}_{\mathrm{L}} \approx 1.50\right.$ at $\left.\lambda_{0}=800 \mathrm{~nm}\right)$ layers by $0.185 \mathrm{~nm} / \mathrm{s}$, respectively. The $\mathrm{HfO}_{2} / \mathrm{SiO}_{2}$ layer stacks are deposited on $1 \mathrm{~mm}$ thick fused silica substrates (1inch diameter) of standard quality. The thickness of each layer is controlled online by a broad band optical monitoring system (BBM) [5]. Applying a conventional halogen lamp, installed below the substrate mount, in combination with a fiber optic CCD spectrometer (Avantes), a wavelength range from $420 \mathrm{~nm}$ up to $1050 \mathrm{~nm}$ is covered. A schematical drawing of the setup is presented in figure 3. 


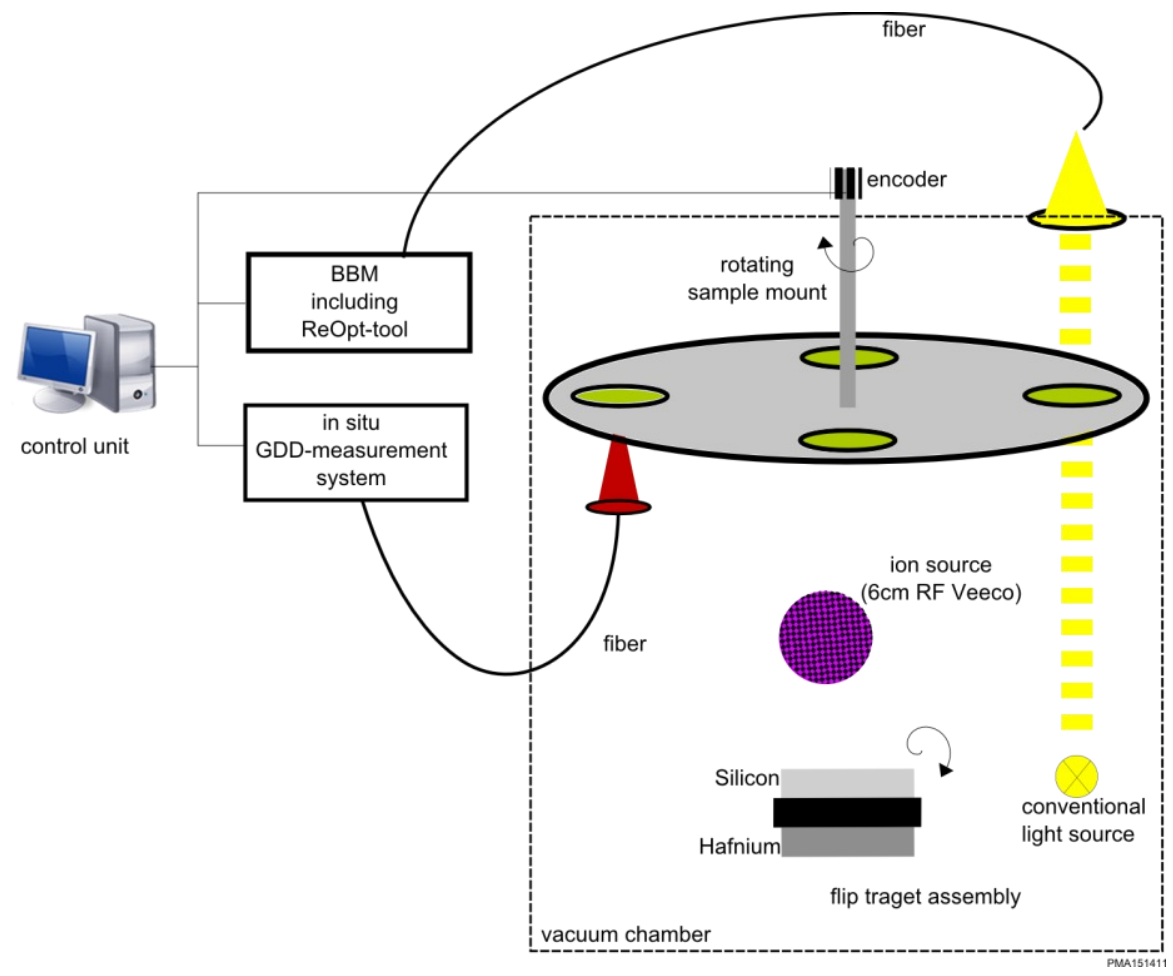

Figure 3. Schematic drawing of the deposition plant. A conventional IBS process is applied for manufacturing $\mathrm{HfO}_{2} / \mathrm{SiO}_{2}$ layer stacks, which are controlled by a BBM using transmittance spectra. Deposition errors are adjusted online by a reoptimization tool (ReOpt) applying the transmission data recorded after termination of each layer. The last layers of the dispersion compensating mirrors are terminated by the in situ group delay dispersion (GDD) measurement system, which mainly consists of a fiber based white light interferometer (WLI) and a fast spectrometer [7].

Possible deposition errors in particular layers can be corrected online during the coating process applying a reoptimization tool [11]. Employing a needle algorithm the subsequent layers can be optimized with respect to the specified group delay dispersion and reflection target values. An optimization cycle can be performed in situ after every coated layer [10]. Especially taking into account the extreme sensitivity of the designed GDD to deposition errors in the last layers, the conventional BBM is used for layer termination until the fifth last layer of the design is finished. Afterwards the deposition process is interrupted and the chamber is vented for aligning the phase interference of the novel in situ white light interferometer (WLI) [7]. Starting the coating process again the last layers are terminated by the described in situ GDD measurement system. In summary the fiber based WLI in combination with a fast high resolution spectrometer displays the current in situ group delay dispersion curve every time the moving sample is passing the collimator head. The detailed setup and principle is presented in [7]. For a precise layer termination the current in situ GDD measurement curve is continuously compared to the theoretic design GDD curve of the particular layer. Figure 4 illustrates the change of the GDD curve during the deposition process. The dashed line represents the calculated GDD curve after the deposition of 72 out of 78 layers. The layer is terminated, if the corresponding in situ measurement (blue solid line) matches best with the calculated one. After a layer change to 73, the deposition process continues with Hafnia as the high index material. The solid lines display an in situ measurement during the deposition in layer 73 approximately $19 \mathrm{~nm}$ before the measurement curve interferes with the estimated design GDD curve (dotted line). 


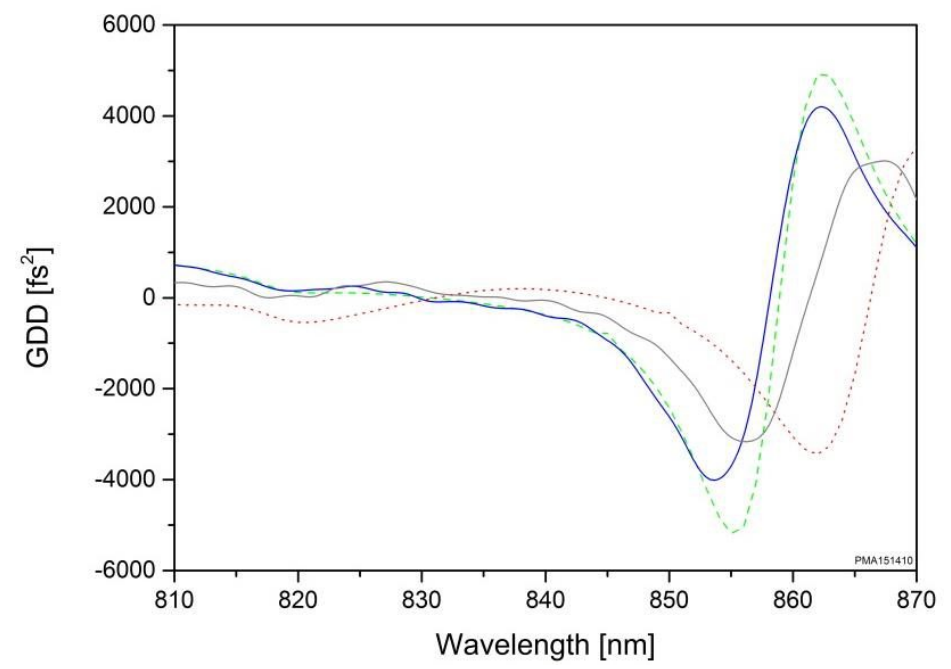

Figure 4. Comparison of in situ WLI measurements to theoretic GDD design curves. The dashed line depicts the theoretic design curve after the deposition of 72 layers, and the dotted one after 73 layers. The solid lines represent in situ WLI measurements during the deposition in layer 73. Layers are terminated in case of a good match between in situ measurement and theoretic design curve of the particular layer.

\section{RESULTS}

Figure 5 depicts the group delay dispersion (GDD) plotted against the wavelength of the NIR target band for the outlined UV dielectric dispersion compensating mirror design after production. For the ex situ GDD measurements a selfconstructed white light interferometer was employed, which is currently operating in a range between $600 \mathrm{~nm}$ up to $1100 \mathrm{~nm}$ in combination with a fast high resolution spectrometer. For the last fifth layers three different samples were produced by the in situ GDD measurement system, which show a similar curve with a maximum deviation of $-50 \mathrm{fs}^{2}$ compared to the theoretic design between $820 \mathrm{~nm}$ up to $860 \mathrm{~nm}$. In comparison the last fifth layers of a reference sample were terminated by a conventional transmission-BBM. Obviously, this monitoring strategy leads to higher deviations and to an oscillating GDD within the target positions compared to the mirror deposited with the in situ GDD layer termination procedure. Regarding the deviation from the theoretic design between $810 \mathrm{~nm}$ up to $870 \mathrm{~nm}$ the samples fabricated with the in situ GDD measurement system exhibit approximately identical sums of error squares of $2.5 \times 10^{7}$. In contrast the sums of error squares of the sample terminated with BBM amounts to a value 1.4 times higher. 


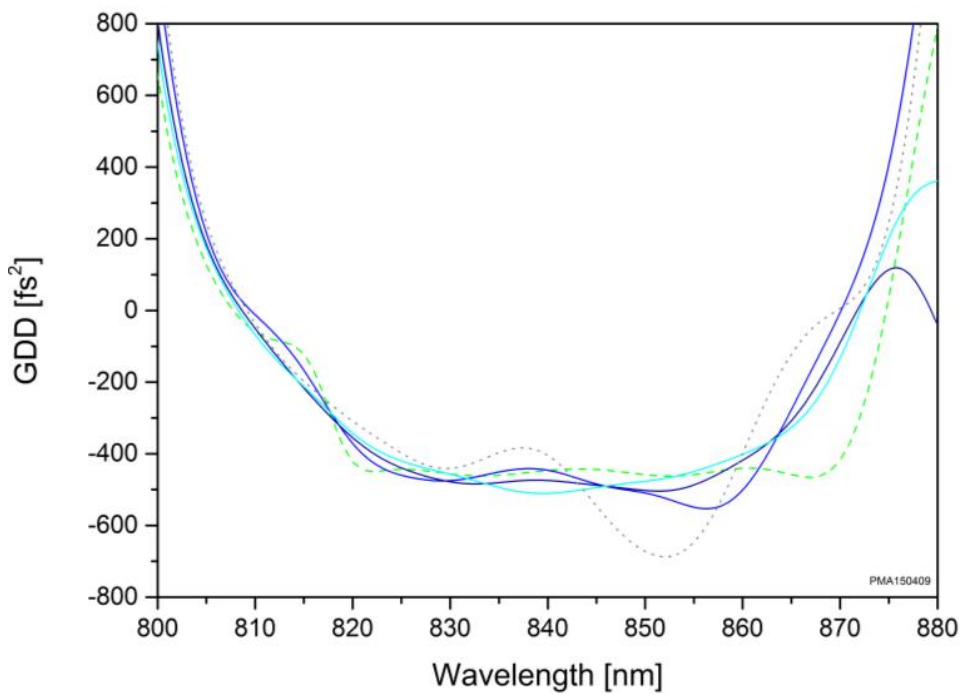

Figure 5. GDD of the manufactured dispersion compensating mirrors (solid lines) comaperd to the estimated design (dashed line) and a reference mirror (dotted line). The mirrors differ in the termination process of the last fifth layers. The solid lines belong to three different samples which were terminated by the novel in situ GDD monitor system. The reference sample was manufactured applying transmittance spectra and termination by a conventional BBM.

In future studies the fabricated samples have to be measured in the UV band, applying ex situ WLI. Currently, reverse engineering, based on the transmission data of the particular terminated by layers, indicates that the GDD band in the NIR and UV responds similar to deposition errors. Figure 6 compares the theoretic to the reversed engineering designs and to the ex situ WLI measurements of the samples terminated by in situ WLI and by a conventional BBM, respectively. Taking figure $6 \mathrm{~b}$ ) into account the reverse engineering points out a maximum GDD deviation of $260 \mathrm{fs}^{2}$ in the NIR band and a deviation of $120 \mathrm{fs}^{2}$ in the UV band for the fabricated sample by conventional BBM. The limited spectral resolution of the spectrometer and the resulting smoothing of the measurement curve is expected to cause deviations in contrast to the reversed engineering curves. Based on the similar behavior to deposition errors, depicted by the reversed engineering to the ex situ WLI measurement curve (see figure 6b), a proper GDD target band is expected in the UV range for the fabricated sample by in situ WLI in figure 6a).

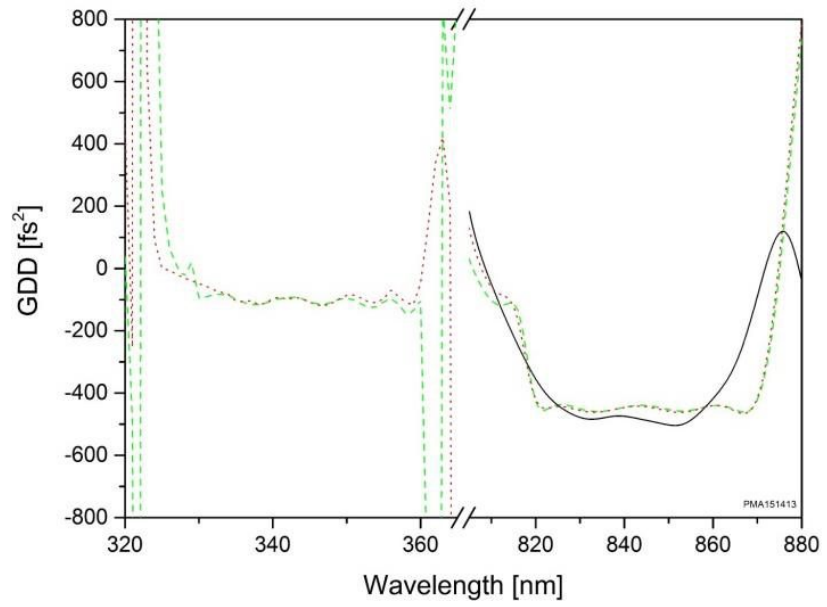

a) Terminated by in situ WLI

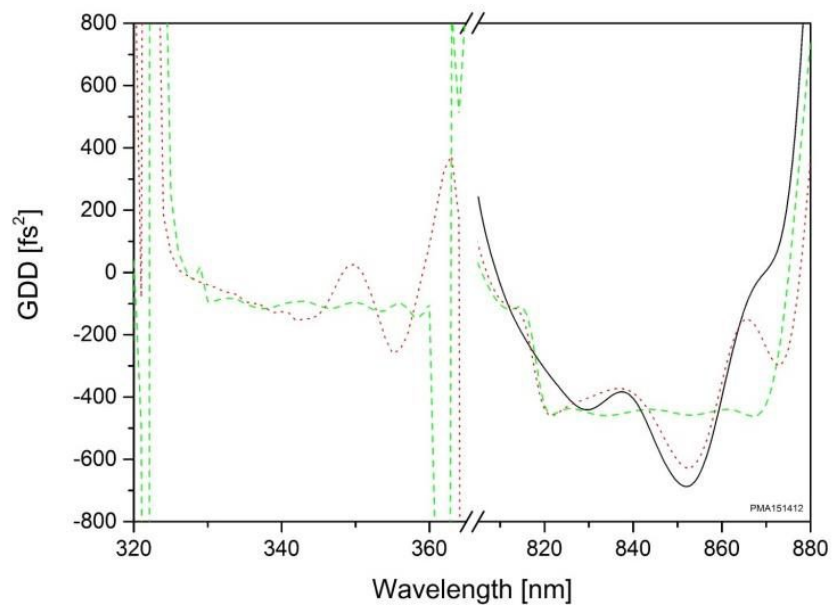

b) Terminated by conventional BBM

Figure 6. Reverse engineering (dotted line) compared to the theoretical design (dashed line) and to the ex situ GDD measurements (solid line) of the particular samples terminated by conventional BBM and in situ WLI. 


\section{CONCLUSION}

In this study a new approach for the precise fabrication of ultra violet dielectric dispersion compensating mirrors is presented. Taking advantage of the in situ group delay dispersion (GDD) measurement system developed by Schlichting et. al. [7], which is actually operating in the near infrared (NIR) spectral range, a design with two target bands is calculated. Ensuring a precise group delay dispersion of the fabricated mirror in the ultra violet (UV) band, while monitoring the (GDD) in the NIR band, an equal sensitivity according to deposition errors in both GDD target bands is required. Due to this demand an empirical design synthesis is implemented, which results in a presented design with an average reflectivity of $94.5 \%$ and a flat GDD of $-100 \mathrm{fs}^{2} \pm 20 \mathrm{fs}^{2}$ between $330 \mathrm{~nm}$ and $360 \mathrm{~nm}$ in the UV range and a GDD of $-450 \mathrm{fs}^{2} \pm 10 \mathrm{fs}^{2}$ between $820-870 \mathrm{~nm}$, respectively. The last layers of the fabricated mirror are terminated by applying the in situ GDD tool. Ex situ GDD measurements of the fabricated mirrors represents a similar GDD curve between $820 \mathrm{~nm}$ up to $860 \mathrm{~nm}$. Reverse engineering, based on the transmission data of the monitored layers, indicates a proper GDD curve in the UV. However, future measurements have to confirm the GDD result in the UV-band.

\section{ACKNOWLEDGEMENT}

This work was partially supported by the Cluster of Excellence "Center of Quantum Engineering and Space-Time Research - QUEST", founded by the German Research Foundation (DFG) and the Federal Ministry of Education and Research (BMBF) within the research project Ultra-LIFE under grant 13N11558.

\section{REFERENCES}

[1] Szipöcs, R., Spielmann, C., Krausz, F., and Ferencz, K., "Chirped multilayer coatings for broadband dispersion control in femtosecond lasers," Optics Letters, 19(3), 201-203 (1994).

[2] Pervak, V., Razskazovskaya, O., Angelov, I. B., Vodopyanov, K. L., and Trubetskov, M., "Dispersive mirror technology for ultrafast lasers in the range 220-4500 nm," Advanced Optical Technologies, 3(1), 55-63 (2014).

[3] Rausch, S., Binhammer, T., Harth, A., Kim, J., Ell, R., Kärtner, F. X., and Morgner, U., "Controlled waveforms on the single-cycle scale from a femtosecond oscillator," Optics Express, 16(13), 9739-9745 (2008).

[4] Rivera, C. A., Bradforth, S. E., and Tempea, G., "Gires-Tournois interferometer type negative dispersion mirrors for deep ultraviolet pulse compression," Optics Express, 18(18), 18615-18624 (2010).

[5] Ristau, D., Ehlers, H., Gross, T., and Lappschies, M., "Optical broadband monitoring of conventional and ion processes," Applied Optics, 45(7), 1495-1501 (2006).

[6] Kärtner, F. X., Matuschek, N., Schibli, T., Keller, U., Haus, H. A., Heine, C., and Tschudi, T., "Design and fabrication of double-chirped mirrors," Optics Letters, 22(11), 831-833 (1997).

[7] Schlichting, S., Willemsen, T., Ehlers, H., Morgner, U. and Ristau, D., "Direct in situ GDD measurement in optical coating process," to be published in this Proc. of SPIE 9627-65, (2015).

[8] Tikhonravov, A.V., and Trubetskov, M.K., "OptiLayer software," http://www.optilayer.com

[9] Pervak, V., Trubetskov, M. K., and Tikhonravov, A. V., "Robust synthesis of dispersive mirrors," Optics Express, 19(3), 2371-2380 (2011).

[10] Amotchkina, T. V., Trubetskov, M. K., Pervak, V., Schlichting, S., Ehlers, H., Ristau, D., and Tikhonravov, A. V., "Comparison of algorithms used for optical characterization of multilayer optical coatings," Applied Optics, 50(20), 3389-3395 (2011).

[11] Schlichting, S., Heinrich, K., Ehlers. H., Ristau, D., "Online re-optimization as a powerful part of enhanced strategies in optical broadband monitoring,” Proc. of SPIE 8168, 81681E (2011). 